Article

\title{
Signal-to-Noise Enhancement of a Nanospring Redox-Based Sensor by Lock-in Amplification
}

\author{
Pavel V. Bakharev and David N. McIlroy * \\ Department of Physics, University of Idaho, Moscow, ID 83844, USA; \\ E-Mail: bakharevpavel@gmail.com \\ * Author to whom correspondence should be addressed; E-Mail: dmcilroy@uidaho.edu; \\ Tel.: +1-208-885-6230.
}

Academic Editor: Michael Tiemann

Received: 27 April 2015 / Accepted: 2 June 2015 / Published: 4 June 2015

\begin{abstract}
A significant improvement of the response characteristics of a redox chemical gas sensor (chemiresistor) constructed with a single $\mathrm{ZnO}$ coated silica nanospring has been achieved with the technique of lock-in signal amplification. The comparison of DC and analog lock-in amplifier (LIA) AC measurements of the electrical sensor response to toluene vapor, at the ppm level, has been conducted. When operated in the DC detection mode, the sensor exhibits a relatively high sensitivity to the analyte vapor, as well as a low detection limit at the $10 \mathrm{ppm}$ level. However, at $10 \mathrm{ppm}$ the signal-to-noise ratio is $5 \mathrm{~dB}$, which is less than desirable. When operated in the analog LIA mode, the signal-to-noise ratio at $10 \mathrm{ppm}$ increases by $30 \mathrm{~dB}$ and extends the detection limit to the ppb range.
\end{abstract}

Keywords: MOS; gas sensor; $\mathrm{ZnO}$; lock-in amplifier

\section{Introduction}

Gas sensors, or chemiresistors, play a critical role in gas and chemical production and high fuel-efficient combustion engines. In addition, they can be used for environmental monitoring [1,2] and to detect hazardous materials, such as explosives vapors [3-9]. Redox-based sensors, or artificial noses, convert chemical information specific to the analyte into analytical electrical signals [4-18]. Hence, they are also excellent scientific tools to analyze molecular interactions at surfaces, be it physisorption or chemisorption. The use of metal oxide nanocrystalline thin films, as well as other more complex 
nano-morphologies, in the capacity of gas sensitive layers in chemiresistors is well documented [4-18]. For metal oxides, the sensing mechanism of the sensors (chemiresistors) is attributed to the depletion, or repletion, of the oxygen at a metal oxide semiconductor (MOS) surface. Consequently, this impacts the depth of the surface depletion layer, thereby producing significant swings in the electrical resistance of a chemiresistor. These studies have demonstrated that a sensor is more responsive to small changes in surface stoichiometry (oxidation state of the metal) if the thickness of the thin film, or the size of the nanostructures, of the MOS layer are comparable to the width of the intrinsic surface depletion layer [4-18].

Gas sensor systems should be able to promptly and reliably identify ("detect" and "recognize") the chemical compound, or compounds, of interest under ambient conditions. One approach to achieve this is to construct a sensor (receptor) array from which a multidimensional electrical response pattern, i.e., a recognition pattern, can be assigned to the compounds. Herein, we present a signal processing methodology using lock-in amplification to increase the signal-to-noise ratio and the absolute detection limit of a single $\mathrm{ZnO}$ coated silica nanospring redox-based gas sensor.

\section{Device Fabrication}

The redox-based gas sensor begins with the growth of a mat of silica nanosprings. The details can be found in previous publications by McIlroy et al. [19] and Wang et al. [20]. The nanosprings are subsequently coated with a $\mathrm{ZnO}$ gas sensitive layer by atomic layer deposition (ALD) [21,22]. Zinc oxide ALD coating of silica nanosprings has demonstrated a several advantageous characteristics compared to other deposition methods, such as chemical vapor deposition (CVD), as well as self-assembled $\mathrm{ZnO}$ nanowire mat $[16,17]$. These advantages include, but are not limited to, the ability to precisely control coating thickness of the gas sensitive layer, the size of the $\mathrm{ZnO}$ nanocrystals, and the ability to uniformly coat complex $3 \mathrm{D}$ structures like nanosprings. $\mathrm{ZnO}$ ALD of insulating $\mathrm{SiO}_{2}$ nanosprings was conducted in a tube furnace maintained at $175{ }^{\circ} \mathrm{C}$ with diethylzinc (DEZn) and deionized water $\left(\mathrm{H}_{2} \mathrm{O}\right)$ as the sources of zinc and oxygen, respectively. During this process, 1 Torr background pressure was maintained in the reaction chamber by a continuous flow $(6 \mathrm{sccm})$ of Ar. An ALD cycle started with a DEZn pulse of $150 \mathrm{~ms}$. This pulse was followed by a $10 \mathrm{~s}$ pressurizing with Ar (up to 2.5 Torr) and an $8 \mathrm{~s}$ pump and an Ar purge. At the next stage, there was a water pulse of $300 \mathrm{~ms}$, followed by another $20 \mathrm{~s}$ pump and an Ar purge. A usual ALD process consisted of 150 cycles at an average $\mathrm{ZnO}$ thickness of $\sim 70 \mathrm{~nm}$.

Two terminal single $\mathrm{ZnO}$ coated nanospring devices (Figure 1a and schematically illustrated in Figure $2 \mathrm{~b}$ ) were fabricated by suspending the $\mathrm{ZnO}$ coated silica nanosprings in isopropyl alcohol (IPA) and transferring the nanospring-alcohol solution onto $25 \mathrm{~mm} \times 25 \mathrm{~mm}$ microscopic glass slides and allowing the IPA to evaporate. The electrical contacts $(50 \mathrm{~nm}$ of Ti layer followed by $150 \mathrm{~nm}$ of Au layer) with $10 \mu \mathrm{m}$ spacing were applied to the glass substrate using standard photolithography and lift-off techniques. SEM image of an individual $\mathrm{ZnO}$ coated nanospring spanning $\mathrm{Ti}-\mathrm{Au}$ electrical contacts is displayed in Figure 1a. Any additional nanosprings lying between the electrodes were removed under an optical microscope using a microprobe needle, thereby ensuring that the electrical response is of a single nanospring. 


\section{Electrical Characterization}

The single nanospring device was mounted to a heating plate to enable temperature regulation. Two electrical probes were used to make electrical contact to the $\mathrm{Ti}-\mathrm{Au}$ pads. The electrical measurements were acquired using a Kiethley 2400 source-sense meter interfaced to a computer via Labview-operated data acquisition software for real time resistance measurements. The single nanospring chemiresistor was placed in a chamber through which a continuous flow of synthetic air $\left(20 \% \mathrm{O}_{2}\right.$ and $\left.80 \% \mathrm{~N}_{2}\right)$ was maintained at all times. Sequential pulses of toluene vapor were generated by the constant flow of Ar gas through a bubbler of liquid toluene and a solenoid valve placed downstream of the bubbler (see Figure 1c). Prior to exposure to toluene vapor, the sensors were allowed to reach a steady state resistance in the synthetic air at atmospheric pressure.
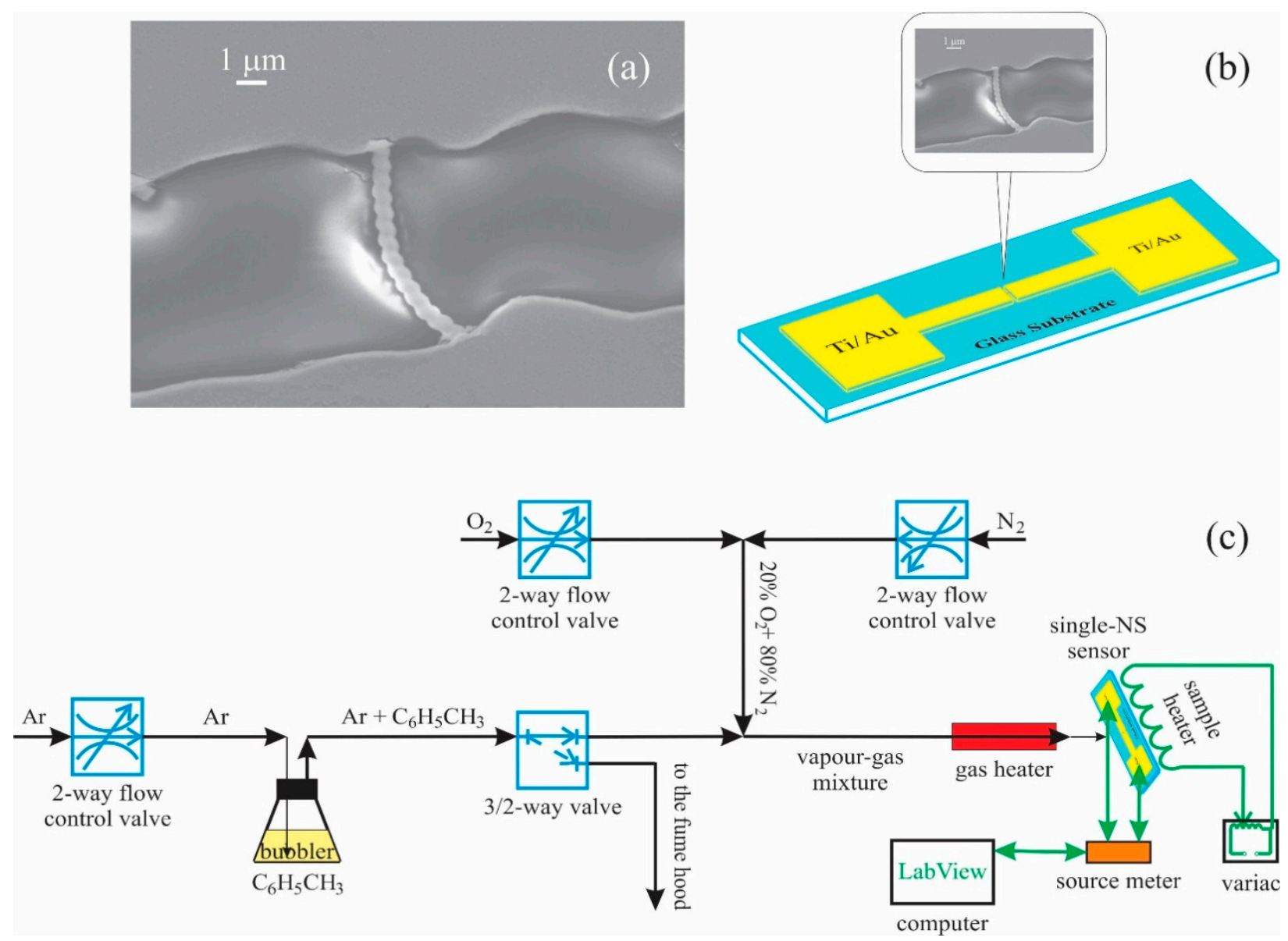

Figure 1. (a) SEM image of a single $\mathrm{ZnO}$ coated nanospring sensor. Schematics (b) of a single $\mathrm{ZnO}$ coated nanospring device and (c) of the two electrode measurement system used to acquire electrical response of the sensor.

The contribution of the glass substrate to the total electrical conductivity of the device was examined by comparing the $\mathrm{I}-\mathrm{V}$ characteristics of the $\mathrm{Ti}-\mathrm{Au}$ terminals with and without the presence of a $\mathrm{ZnO}$ coated nanospring [5]. In the absence of a nanospring, at a temperature of $300^{\circ} \mathrm{C}$, an open circuit with a nominal current (orders of magnitude less than with a nanospring present) was observed, where the nominal current is attributed to residual carbon on the glass surface. Moreover, unlike the single 
nanospring device, the electrodes devoid of $\mathrm{ZnO}$ coated nanospring did not respond to the analyte (toluene) vapor.

The DC electrical responses of the single $\mathrm{ZnO}$ coated nanospring chemiresistor operated at $310^{\circ} \mathrm{C}$ to toluene partial vapor pressures of $60 \mathrm{ppm}, 40 \mathrm{ppm}, 20 \mathrm{ppm}, 10 \mathrm{ppm}$ are shown in Figure 2. The chemiresistor exhibits good linearity of the calibration graph (response vs analyte concentration) with a slope of $18 \%$ response change per 40 ppm toluene partial pressure change.
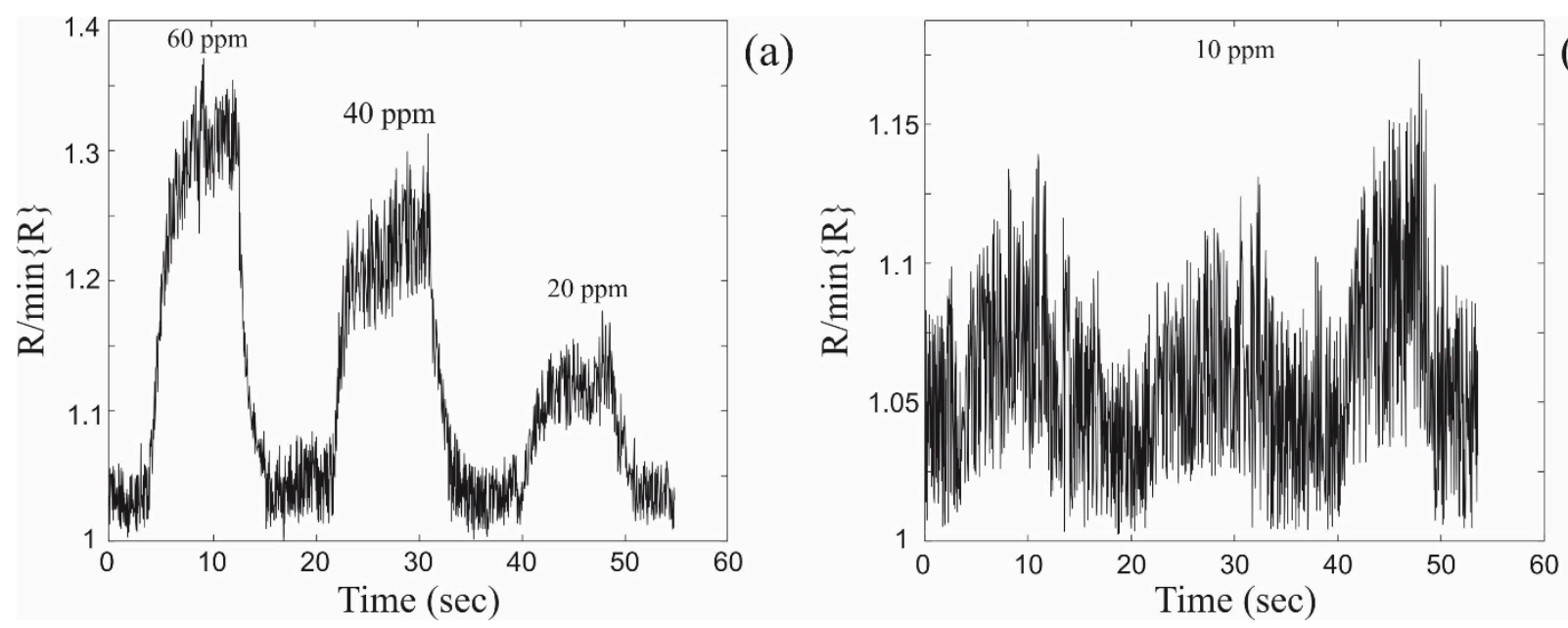

Figure 2. DC electrical response of a single $\mathrm{ZnO}$ coated nanospring sensor. The relative changes in resistance upon exposure to toluene vapor at (a) $60 \mathrm{ppm}, 40 \mathrm{ppm}, 20 \mathrm{ppm}$ and (b) 10 ppm.

The overall noise in the electrical signal illustrated in Figure 2 has multiple sources and, in general, can be subdivided into intrinsic and extrinsic noise sources.

There are many sources of intrinsic noise, each having unique characteristics. For DC measurements conducted for the single $\mathrm{ZnO}$ coated nanospring device, the overall noise is comprised of three main constituents, which are thermal noise (Johnson-Nyquist noise), shot noise, and flicker $(1 / f)$ noise $[23,24]$. Thermal noise is the electronic noise generated by the thermal equilibrium fluctuations of charge carriers. This noise type does not depend on applied voltage. It appears as white noise with power density spectrum given by,

$$
S_{\mathrm{Th}}=4 k_{\mathrm{B}} T_{\mathrm{NS}} R
$$

where $k_{\mathrm{B}}$ is the Boltzmann constant, $T_{\mathrm{NS}}$ is the absolute temperature of the sensor, and $R$ is the resistance of the individual $\mathrm{ZnO}$ coated nanospring. Heating of the $\mathrm{ZnO}$ thermally activates surface sites (such as oxygen vacancies), which subsequently chemisorb atmospheric oxygen. This, in turn, increases the sensor resistance $R$. Hence, according to Equation (1), at elevated temperatures the thermal noise prevails over other intrinsic noise sources.

With that said, shot noise and flicker $(1 / f)$ noise can become dominant sources of noise at low temperatures with diminished thermal noise [5]. Shot noise is a white noise source associated with the

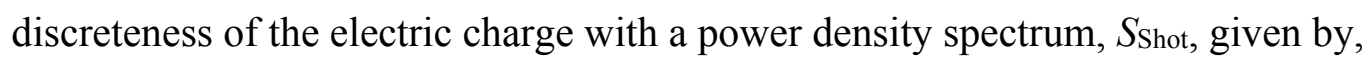

$$
S_{\text {Shot }}=2 e I
$$


where $e$ is the electron charge and $I$ is the average current in the device. Shot noise can be observed in electronic devices with internal potential barriers, such as potential barriers formed at crystal grain interfaces and Schottky contacts.

Two major theories have been developed to explain the physical origin of flicker $(1 / f)$ noise in MOS devices, namely, the number fluctuation theory (NFT) [25-27] originally proposed by McWhorter [28] and the bulk mobility fluctuation theory (MFT) based on Hooge's model [29]. According to the NFT, flicker noise arises from random trapping and detrapping of carriers at defects near the MOS surface and near the MOS/insulator interface $\left(\mathrm{ZnO} / \mathrm{SiO}_{2}\right.$ interface in this study). Hence, this type of noise can be observed mainly in nanoscale MOS devices with a high surface-to-volume ratio, which is the case for $\mathrm{ZnO}$ coated nanosprings. The mobility fluctuation theory attributes the $1 / f$ noise to fluctuations in bulk mobility caused by phonon scattering. According to Hooge's empirical relation, the power density spectrum $S_{\mathrm{Fl}}$ can be expressed as

$$
S_{\mathrm{Fl}}=\alpha_{\mathrm{H}} \frac{V^{2}}{N f}
$$

where $N$ is the total number of carriers in the semiconductor, $V$ is the applied bias voltage, and $\alpha_{H}$, known as Hooge's parameter, is an empirical constant used as the measure of the noise magnitude [29].

Extrinsic noise sources include electromagnetic fields that couple into sensitive circuit (radiative coupling, capacitive coupling, inductive coupling, and conductive coupling), mechanical vibrations that trigger piezoelectric materials such as $\mathrm{ZnO}$ thin films [30,31] to generate unintended $\mathrm{AC}$ electrical signals, or in the present case, noise from random fluctuations in concentrations of flowing gases and vapors.

In order to significantly increase the signal-to-noise ratio (SNR) and subsequently extend the lower detection limit of a $\mathrm{ZnO}$-coated silica nanospring chemiresistor/sensor, the analog lock-in amplifier (LIA) technique has been integrated into the signal processing of the sensor signal. The inclusion of LIA changes the operating mode of the sensor from DC to AC through the introduction of a modulated input signal generated by a function generator. The AC electrical response measurements were carried out using a Stanford Research Systems SR510 analog LIA. The measurement scheme of the lock-in based experimental set-up used to detect small amplitude modulations in AC signals of the single $\mathrm{ZnO}$ coated nanospring chemiresistor is shown in Figure $3 \mathrm{a}$.

A layout of noisy signal pathway through an analog LIA is illustrated in Figure $3 \mathrm{~b}$. An input time-dependent signal $s\left(t ; \omega_{0}\right)$ in the presence of noise $n(t)$ is multiplied with the reference analog LIA waveform $\operatorname{Sref}\left(t ; \omega_{0}\right)$ at the multiplier, also known as the Phase-Sensitive Detector (PSD). PSD generates a signal, the DC component of which is proportional to the amplitude of the AC input signal $s(t)$ and depends on the phase difference between the input signal and the reference signal. The DC component in the PSD output signal can be extracted by means of the low-pass filter (LPF) with the transfer function $H(\omega)$ and the characteristic band-width $\Delta \omega_{\mathrm{LPF}}$. The lock-in quality factor Q can be expressed as,

$$
\mathrm{Q}=\frac{\omega_{0}}{\Delta \omega_{\mathrm{LPF}}}
$$

The SNR can be defined as the power ratio of a signal to the background noise: 


$$
\mathrm{SNR}=\frac{\overline{P_{s}(t)}}{\overline{P_{n}(t)}}
$$

where $\overline{P_{S}(t)}$ and $P_{n}(t)$ are the average spectral powers of the signal and the background noise, respectively. In decibel scale, the SNR is given as,

$$
\mathrm{SNR}_{\mathrm{dB}}=10 \log _{10}(\mathrm{SNR})
$$

The mean square value of the noise amplitude should be used to estimate the experimental SNR, since the background noise is a random signal with zero mean value.

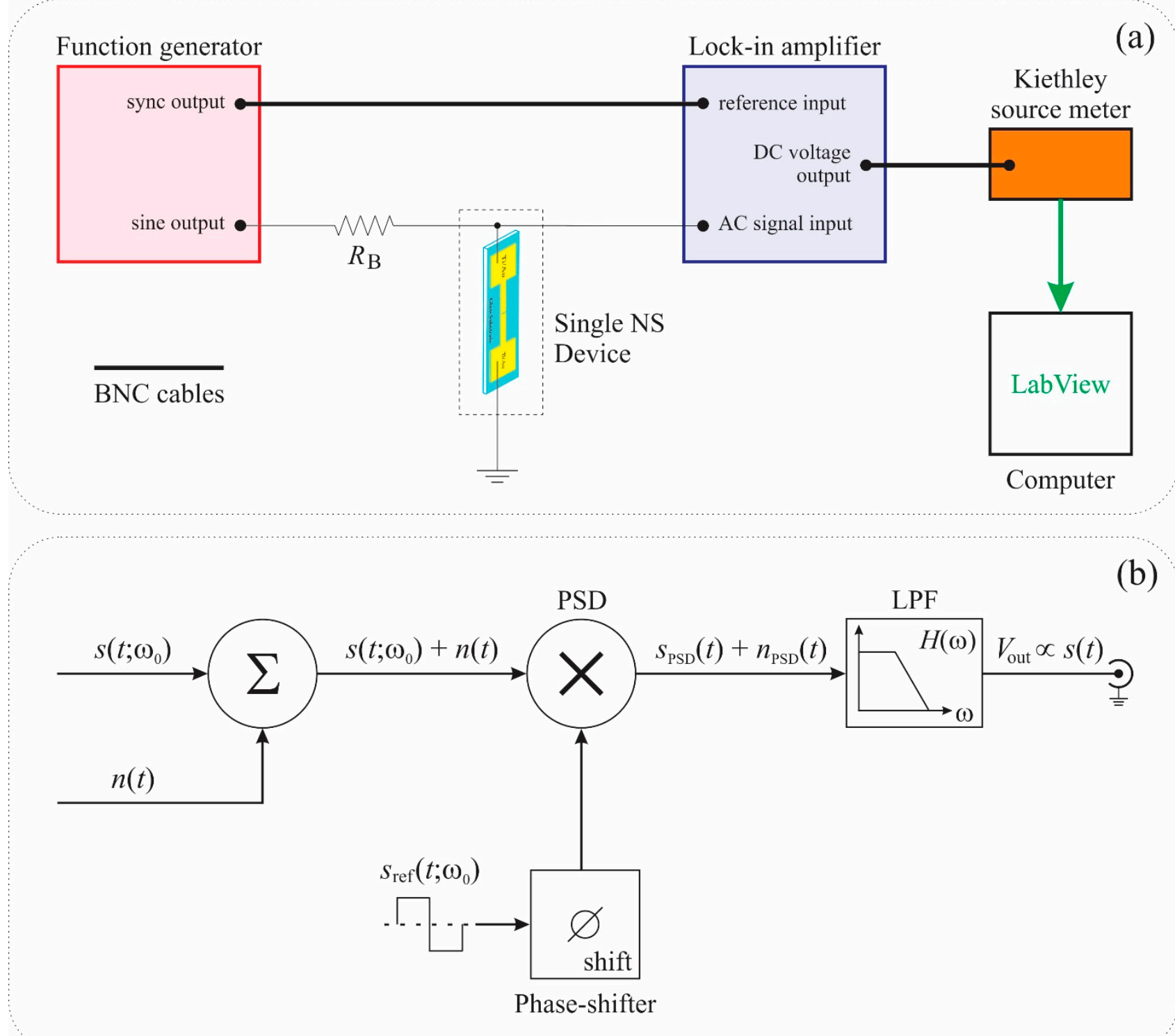

Figure 3. (a) Schematic of the lock-in based experimental set-up utilized for toluene vapor detection; (b) Layout of signal pathway through an analog lock-in amplifier (LIA). The symbols $\Sigma$ and $\times$ represent summing and mixing (multiplying) stages, respectively. Phase-Sensitive Detector (PSD) and low-pass filter (LPF) are a multiplier, called the phase sensitive detector, and a low-pass filter with transfer function $H(\omega)$, respectively. 
The noise obtained from the DC measurements, as well as the noise at the LIA input $n(t)$ with power spectral density $N(\omega)$, can be represented as the following limit:

$$
n(t)=\lim _{\Delta \omega \rightarrow 0} \sum_{i=-\infty}^{+\infty} C_{i} \sin \left(\omega_{i} t+\theta_{i}\right) ; \omega_{i}=i \Delta \omega
$$

where $C_{i}$ is the Fourier coefficient. Upon the transition through the LIA with the low-pass filter (LPF) transfer function $H(\omega)$, the mean-square value of the output noise is,

$$
\overline{n_{\text {out }}^{2}(t)}=\frac{1}{2 \pi} \int_{-\infty}^{+\infty} N_{\text {PSD }}(\omega)|H(\omega)|^{2} d \omega
$$

where $N_{\operatorname{PSD}}(\omega)$ is the power spectral density of the noise upon passage through the PSD. The transfer function, $H(\omega)$, of the $R C$ low-pass filter (LPF) is,

$$
H(\omega)=\frac{1}{1+i \omega \tau_{R C}}
$$

where $\tau_{R C}$ is a characteristic time constant of the LPF. Note, the goal of this work is not to identify the dominant noise mode of the chemiresistor, but to increase the SNR. Identification of the degree to which the different types of noise contribute to the signal will be the subject of a future study.

The electrical response of a single $\mathrm{ZnO}$ coated nanospring chemiresistor measured at LIA output to toluene vapor pulses at $60 \mathrm{ppm}, 40 \mathrm{ppm}, 20 \mathrm{ppm}$ and $10 \mathrm{ppm}$ is displayed in Figure 4. The LIA chemiresistor signals in Figure 4 are superior to the DC signals in Figure 2. The contribution of noise to the signal of the chemiresistor in LIA mode is practically negligible down to $20 \mathrm{ppm}$. At $10 \mathrm{ppm}$ of toluene the signal of the chemiresistor operated in the DC mode is not trustworthy. By contrast, when operated in LIA mode, the chemiresistor signal at $10 \mathrm{ppm}$ is as reliable as that at $60 \mathrm{ppm}$. Note, there is no loss of information with regards to the amplitude of the signal when operated in the LIA mode. The utilization of the LIA technique significantly improves not only the electrical response characteristics of a single $\mathrm{ZnO}$ coated nanospring sensor (receptor), but also considerably expands the recognition capabilities of the gas sensor array (electronic nose) in the frames of linear discrimination analysis (LDA), independent component analysis (ICA), principal component analysis (PCA) and other multiple odor recognition methods $[9,11,32-34]$. The repeatability of the signal profile and intensity will greatly increase the reliability of LDA-based detection.

A summary SNR of the chemiresistor operated in DC and LIA modes is presented in Figure 5, which clearly demonstrates the significant increase in the SNR at all concentrations upon signal passage through the LIA. This effect is attributed to the fact that only the low-frequency components of the noise power spectral density, $N_{\mathrm{PSD}}(\omega)$, contribute to the LIA output noise within its equivalent noise bandwidth (ENBW) $\Delta \omega_{\mathrm{LPF}}$. The ENBW, $\Delta \omega_{\mathrm{LPF}}$, of the $R C \mathrm{LPF}$ is approximately equal to $1 / \tau_{\mathrm{RC}}$. For practical purposes of gas sensors, real-time response measurements are required, so the integration timescale of LIA should be low enough to facilitate relatively short detection response times. Hence, the characteristic time constant, $1 / \tau_{\mathrm{RC}}$, of the $R C \mathrm{LPF}$ was set to be $50 \mathrm{~ms}$ since it is much shorter than the sensor response time [5,6] and simultaneously very long compared to the period, $T_{0}$, of the reference signal ( $T_{0}=10 \mathrm{~ms}$ ). Comparing the SNRs of the DC and LIA measurements performed for a single $\mathrm{ZnO}$ coated silica nanospring, the utilization of the conventional analog LIA leads to the sensor resolution reduction by a factor of 20 . 


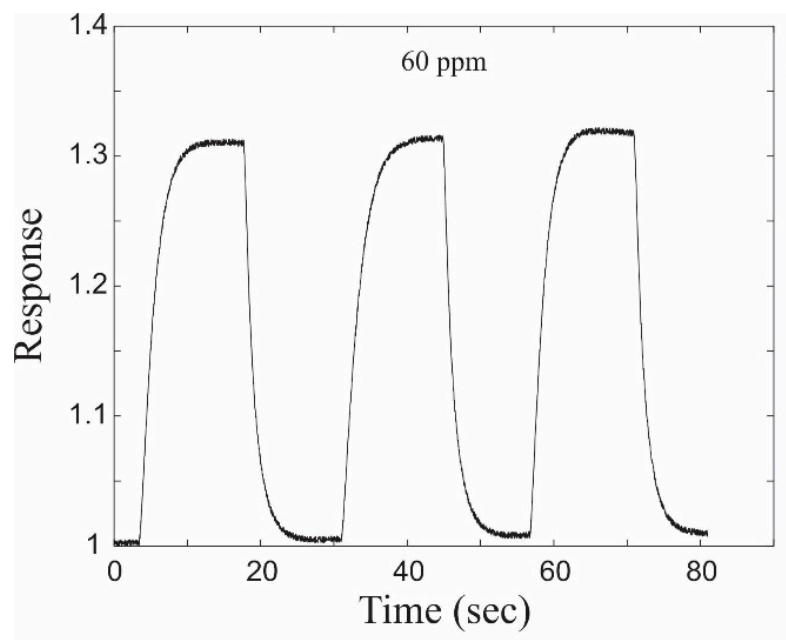

(a)
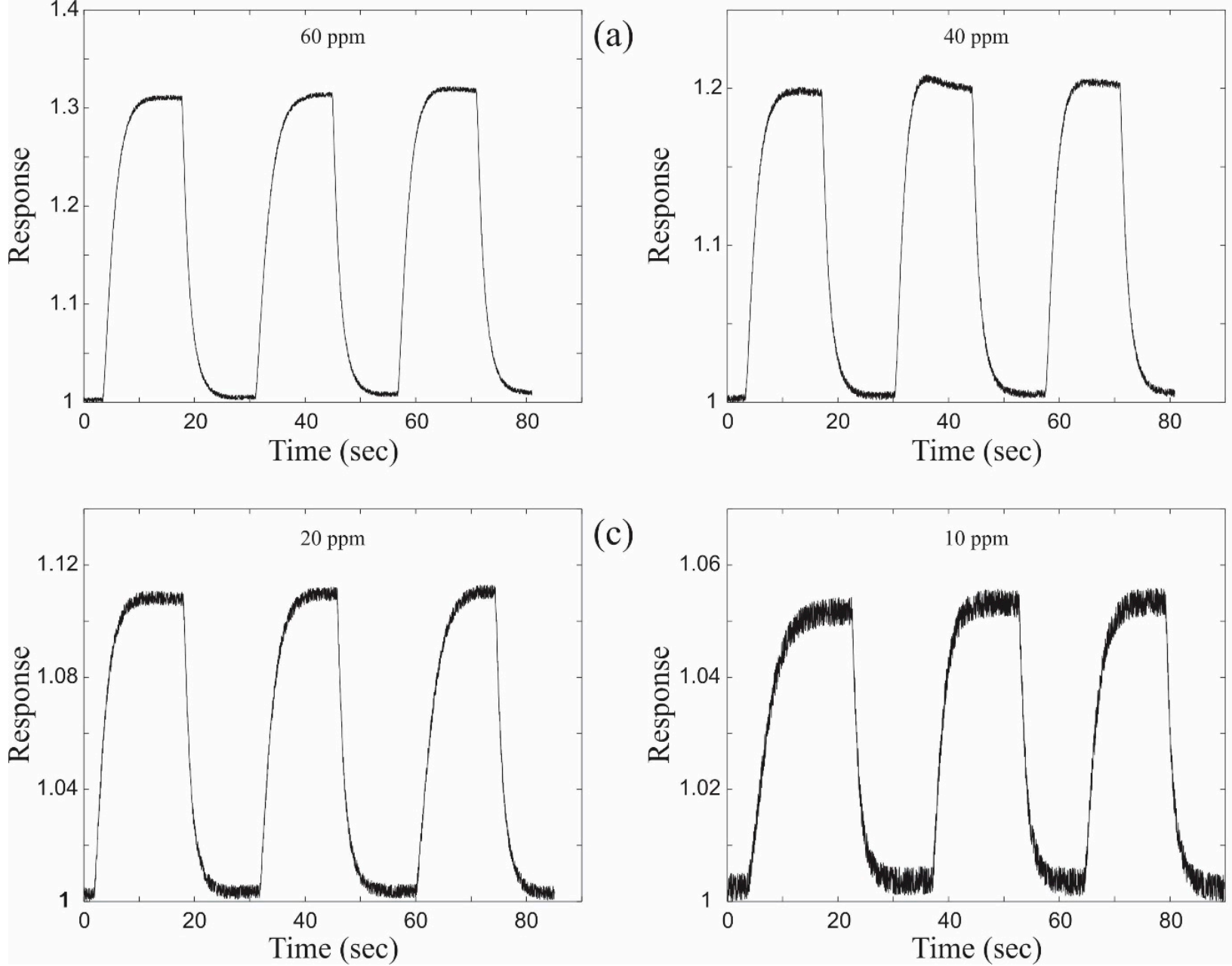

(c)

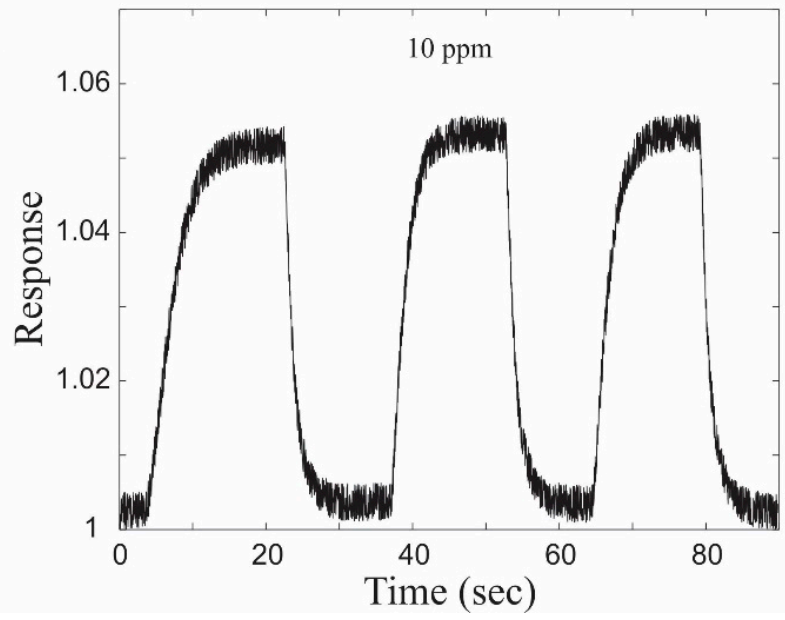

(d)

Figure 4. The electrical response of a single $\mathrm{ZnO}$ coated nanospring device measured at lock-in amplifier (LIA) output upon exposure to toluene vapor at (a) 60 ppm; (b) 40 ppm; (c) $20 \mathrm{ppm}$ and (d) $10 \mathrm{ppm}$.

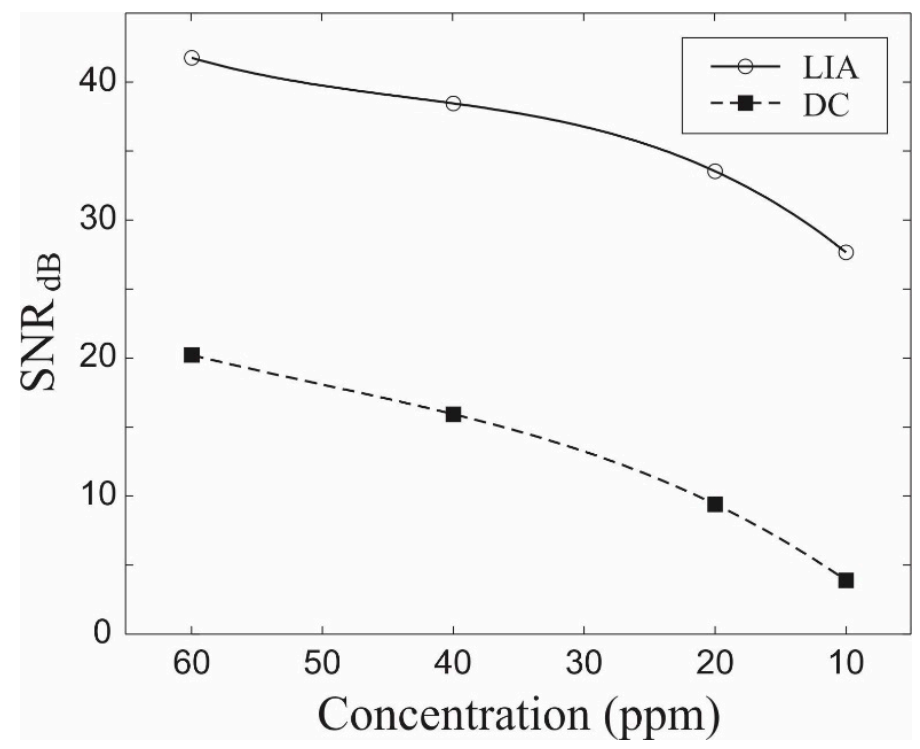

Figure 5. Signal-to-noise ratios (SNRs) in decibel scale of a single $\mathrm{ZnO}$ coated nanospring sensor obtained by utilizing the DC and the analog lock-in amplifier (LIA) modes of detection. 
Hence, the analog LIA measurements of small AC modulation signals generated by the redox and the analyte oxidation processes at the $\mathrm{ZnO}$ surface [5-9] enables a detection limit of the sensor to be reduced from tens of ppm (for DC measurements) to tens of ppb range under equivalent experimental conditions.

\section{Conclusions}

A new nanomaterials-based chemical sensor (chemiresistor) has been developed that has excellent sensitivity and SNR. The chemiresistor has been constructed with a single $\mathrm{ZnO}$ coated silica nanospring. The gas sensor response to the analyte (toluene) vapor is attributed to its catalytic oxidation of the analyte at the $\mathrm{ZnO}$ surface, hence creating an oxygen deficient surface of $\mathrm{ZnO}$, and self-refreshing through dissociative chemisorption of oxygen (redox process). DC mode chemiresistor responses to pulses of toluene vapor at the ppm concentration levels demonstrate high sensitivity and the detection limit of $10 \mathrm{ppm}$ of the single $\mathrm{ZnO}$ coated nanospring chemiresistor. By comparison, when the nanospring chemiresistor is operating in the analog lock-in amplifier mode a superior SNR is achieved and the detection limit is extended to tens of ppb. In conclusion, it has been shown that the operation of a chemiresistor operated in the analog LIA mode significantly increases the SNR, improves sensitivity and considerably lowers the detection limit. The LIA operating mode can be used with essentially any chemiresistor, or other types of chemical sensors, especially when employing LDA and ICA signal recognition.

\section{Acknowledgments}

Authors would like to thank the Office of Naval Research (grant \# N00014-10-1-0282) for supporting this work. DMc would like to acknowledge the USDA and the Dyess Faculty Fellowship for their support.

\section{Author Contributions}

Pavel Bakharev is the main author of the current paper. In addition to writing the paper, he performed fabrication of the sensor device (VLS synthesis of silica nanosprings, ALD coating of a nanospring mat with $\mathrm{ZnO}$, fabrication of the single $\mathrm{ZnO}$ coated nanospring sensor by photolithography in a clean room of class 1000) and conducted its electrical characterization. Pavel Bakharev constructed and calibrated the ALD system, which is used for $\mathrm{ZnO}$ deposition, and assembled the gas sensor test station for DC and lock-in amplifier based AC measurements. David Mcllroy is the academic advisor of Pavel Bakharev, who managed the research process, the laboratory facilities and edited the current paper.

\section{Conflicts of Interest}

The authors declare no conflict of interest.

\section{References}

1. Sekhar, P.; Brosha, E.; Mukundan, R.; Garzon, F. Chemical sensors for environmental monitoring and homeland security. ECS 2010, 19, 35-40. 
2. Pejcic, B.; Eadington, P.; Ross, A. Environmental monitoring of hydrocarbons: a chemical sensor perspective. Environ. Sci. Tech. 2007, 41, 6333-6342.

3. Bielecki, Z.; Janucki, J.; Kawalec, A.; Mikolajczyk, J.; Palka, N.; Pasternak, M.; Pustelny, T.; Stacewicz, T.; Wojtas, J. Sensors and systems for the detection of explosive devices-An overview. Metrol. Meas. Syst. 2012, 19, 3-28.

4. Procek, M.; Stolarczyk, A.; Pustelny, T.; Maciak, E. A Study of a QCM Sensor Based on $\mathrm{TiO}_{2}$ Nanostructures for the Detection of $\mathrm{NO}_{2}$ and Explosives Vapours in Air. Sensors 2015, 15, 9563-9581.

5. Bakharev, P.; McIlroy, D. The effect of the periodic boundary conditions of a ZnO-coated nanospring on its surface redox-induced electrical response. Nanotechnology 2014, 25, 475501.

6. Bakharev, P.; Dobrokhotov, V.; McIlroy, D. A method for integrating ZnO coated nanosprings into a low cost redox-based chemical sensor and catalytic tool for determining gas phase reaction kinetics. Chemosensors 2014, 2, 56-68.

7. Dobrokhotov, V.; Oakes, L.; Sowell, D.; Larin, A.; Hall, J.; Kengne, A.; Bakharev, P.; Corti, G.; Cantrell, T.; Prakash, T.; et al. ZnO coated nanospring-based chemiresistor. J. Appl. Phys. 2012, 111, 044311:1-044311:8.

8. Dobrokhotov, V.; Oakes, L.; Sowell, D.; Larin, A.; Hall, J.; Barzilov, A.; Kengne, A.; Bakharev, P.; Corti, G.; Cantrell, T.; et al. Thermal and optical activation mechanisms of nanospring-based chemiresistors. Sensors 2012, 12, 5608-5622.

9. Dobrokhotov, V.; Oakes, L.; Sowell, D.; Larin, A.; Hall, J.; Kengne, A.; Bakharev, P.; Corti, G.; Cantrell, T.; Prakash, T.; et al. Toward the nanospring-based artificial olfactory system for trace-detection of flammable and explosive vapors. Sens. Actuators B Chem. 2012, 168, 138-148.

10. Sysoev, V.; Goschnick, J.; Schneider, T.; Strelcov, E.; Kolmakov, A. A gradient microarray electronic nose based on percolating $\mathrm{SnO}_{2}$ nanowire sensing elements. Nano Lett. 2007, 7, 3182-3188.

11. Bochenkov, V.; Sergeev, G. Bochenkov, V.; Sergeev, G. Metal Oxide Nanostructures and Their Applications, 2nd ed.; Umar, H., Ed.; American Scientific Publishers: Valencia, CA, USA, 2010; Volume 3, pp. 31-52.

12. Liu, X.; Cheng, S.; Liu, H.; Hu, S.; Zhang, D.; Ning, H. A survey on gas sensing technology. Sensors 2012, 12, 9635-9665.

13. Barsan, N.; Weimar, U. Conduction model of metal oxide gas sensors. J. Electroceram. 2001, 7, $143-167$.

14. Barsan, N.; Weimar, U. Understanding the fundamental principles of metal oxide based gas sensors; the example of $\mathrm{CO}$ sensing with $\mathrm{SnO}_{2}$ sensors in the presence of humidity. J. Phys. Condens. Matter 2003, 15, R813-R839.

15. Gurlo, A.; Bârsan, N.; Oprea, A.; Sahm, M.; Sahm, T.; Weimar, U. An n- to p-type conductivity transition induced by oxygen adsorption on a-Fe2 $\mathrm{O}_{3}$. Appl. Phys. Lett. 2004, 85, doi:10.1063/1.1794853.

16. Ra, H.; Khan, R.; Kim, J.; Kang, B.; Im, Y. The effect of grain boundaries inside the individual $\mathrm{ZnO}$ nanowires in gas sensing. Nanotechnology 2010, 21, doi:10.1088/0957-4484/21/8/085502.

17. Li, Q.; Gao, T.; Wang, Y.; Wang, T. Adsorption and desorption of oxygen probed from $\mathrm{ZnO}$ nanowire films by photocurrent measurements. Appl. Phys. Lett. 2005, 86, 123117:1-123117:3. 
18. Gas'kov, A.; Rumyantseva, M. Nature of gas sensitivity in nanocrystalline metal oxides. J. Appl. Chem. 2001, 74, 440-444.

19. McIlroy, D.; Alkhateeb, A.; Zhang, D.; Aston, D.; Marcy, A.; Norton, M. Nanospring formation-Unexpected catalyst mediated growth. J. Phys. Condens. Matter 2004, 16, R415-R440.

20. Wang, L.; Major, D.; Paga, P.; Zhang, D.; Norton, M.; McIlroy, D. High yield synthesis and lithography of silica-based nanospring mats. Nanotechnology 2006, 17, S298-S303.

21. George, S.; Ott, A.; Klaus, J. Surface chemistry for atomic layer growth. J. Phys. Chem. 1996, 100, 13121-13131.

22. Guziewicz, E. ZnO by ALD-advantages of the material grown at low temperature. Acta Phys. Pol. A 2009, 116, 814-817.

23. Seguin, J.-L.; Gomri, S.; Guerin, J.; Aguir, K. Bases of noise spectroscopy for enhancing metallic oxide gas sensors selectivity. In Science and Technology of Chemiresistor Gas Sensors; Aswal, K.D., Gupta, S.K., Eds.; Nova Science Publishers Inc.: New York, NY, USA, 2007; pp. 351-371.

24. Konczakowska, A.; Wilamowski, B. Noise in semiconductor devices. In Fundamentals of Industrial Electronics, 2nd ed.; CRC Press: Boca Raton, FL, USA, 2011.

25. Christensson, S.; Lundstrom, I.; Svensson, C. Low frequency noise in MOS transistors-I theory. Solid-State Electron. 1968, 11, 797-812.

26. Bertz, F. Theory of low frequency noise in Si MOST's. Solid-State Electron. 1970, 13, 631-647.

27. Hsu, S. Surface state related 1/f noise in MOS transistors. Solid-State Electron. 1970, 13, 1451-1459.

28. McWhorter A 1/f noise and germanium surface properties. In Semiconductor Surface Physics; University of Philadelphia Press: Philadelphia, PA, USA, 1957; p. 207.

29. Hooge, F. 1/f noise. Physica 1976, 83B, 14.

30. Fan, Z.; Lu, J.G. Zinc oxide nanostructures: synthesis and properties. J. Nanosci. Nanotechnol. 2005, 10, 1561-1573.

31. Kanno, I.; Kotera, H.; Wasa, K. Measurement of transverse piezoelectric properties of PZT thin films. Sens.Actuators A Phys. 2003, 107, 68-74.

32. Sysoev, V.; Strelcov, E.; Kolmakov, A. Multidimensional micro-arrays based on metal oxide nanowires for electronic nose application. In Integrated Analytical Systems; Springer: New York, NY, USA, 2013; pp. 465-502.

33. Tripathy, A.; Mohanty, A.; Mohanty, M. Electronic nose for black tea quality evaluation using kernel based clustering approach. Int. J. Image Process. 2012, 6, 86-93.

34. Brudzewski, K.; Osowski, S.; Dwulit, A. Recognition of coffee using differential electronic nose, IEEE Trans. Instrum. Meas. 2012, 61, 1803-1810.

(C) 2015 by the authors; licensee MDPI, Basel, Switzerland. This article is an open access article distributed under the terms and conditions of the Creative Commons Attribution license (http://creativecommons.org/licenses/by/4.0/). 